\title{
Extrusion and Characterization of Functionalized Cellulose Whisker \\ Reinforced Polyethylene Nanocomposites
}

Aparecido Junior de Menezes $^{1}$, Gilberto Siqueira ${ }^{1}$, Aprigio A. S. Curvelo ${ }^{2}$ and Alain Dufresne ${ }^{1 *}$

${ }^{1}$ Grenoble Institute of Technology, The International School of Paper, Print Media and Biomaterials (PAGORA), BP65, 38402 Saint Martin d'Hères cedex, France

${ }^{2}$ Instituto de Química de São Carlos (IQSC), Universidade de São Paulo (USP), C.P. 780, 13560-970 São Carlos, Brazil.

*E-mail: Tel: +33 4768269 95; Fax: +33 4768269 33; Alain.Dufresne @ efpg.inpg.fr

\begin{abstract}
The surface of ramie cellulose whiskers has been chemically modified by grafting organic acid chlorides presenting different lengths of the aliphatic chain by an esterification reaction. The occurrence of the chemical modification was evaluated by FTIR and X-ray photoelectron spectroscopies, elemental analysis and contact angle measurements. The crystallinity of the particles was not altered by the chain grafting, but it was shown that covalently grafted chains were able to crystallize at the cellulose surface when using $\mathrm{C} 18$. Both unmodified and functionalized nanoparticles were extruded with low density polyethylene to prepare nanocomposite materials. The homogeneity of the ensuing nanocomposites was found to increase with the length of the grafted chains. The thermomechanical properties of processed nanocomposites were studied by differential scanning calorimetry (DSC), dynamical mechanical analysis (DMA) and tensile tests. A significant improvement in terms of elongation at break was observed when sufficiently long chains were grafted on the surface of the nanoparticles. It was ascribed to improved dispersion of the nanoparticles within the LDPE matrix.
\end{abstract}

Keywords: Cellulose; Whiskers; Nanocomposites; Grafting 


\section{Introduction}

Over the last two decades a good deal of work has been dedicated to the use of lignocellulosic fibers as reinforcing elements in polymeric matrix and for the possibility of replacing conventional fibers such as glass by natural fibers in reinforced composites [1]. However, one of the main drawbacks of lignocellulosic fibers, among others, is the important variation of properties inherent to any natural product. Indeed, their properties are related to climatic conditions, maturity, and type of soil. Disturbances during plant growth also affect the plant structure and are responsible for the enormous scatter of mechanical plant fiber properties.

One of the basic idea to achieve further improved fiber and composite is to eliminate the macroscopic flaws by disintegrating the natural grown fibers, and separating the almost defect free highly crystalline fibrils. Indeed, natural fibers display a hierarchical structure and present a multi-level organization. In fact, cellulose chains are biosynthesized by enzymes and aggregate to form microfibrils. Depending on their origin, the microfibril section ranges between 2 and $20 \mathrm{~nm}$ for lengths that can reach several tens of microns. They aggregate further to form fibers. Therefore, each natural fiber can be considered as a string of cellulose crystallites, linked along the chain axis by disordered domains.

Aqueous suspensions of cellulose nanocrystals can be prepared by acid hydrolysis of the biomass. The object of this treatment is to dissolve away regions of low lateral order so that the water-insoluble, highly crystalline residue may be converted into a stable suspensoid by subsequent vigorous mechanical shearing action. The resulting nanocrystals occur as rod-like particles or whiskers, which dimensions depend on the nature of the substrate, but range in the nanometer scale. Because these whiskers contain only a small number of defects, their axial Young's modulus is close to the one derived from theoretical chemistry and potentially stronger than steel and similar to Kevlar. It has been first experimentally determined in 1962 and a value of $137 \mathrm{GPa}$ was reported [2]. This value differs from the theoretical estimate of 167.5 GPa reported by Tashiro and Kobayashi [3]. More recently, Raman spectroscopy technique has been used to measure the elastic modulus of native cellulose crystals. A value around $143 \mathrm{GPa}$ has been reported [4]. These highly stiff nanoparticles are therefore suitable for the processing of green nanocomposite materials.

Then, the main problem is related to the homogeneous dispersion of these nanoparticles within a polymeric matrix. Because of the high stability of aqueous suspensions of cellulose whiskers, water is the preferred processing medium. Hydrosoluble polymers are therefore well adapted for the processing of cellulose whiskers reinforced nanocomposites [5]. Solid nanocomposite films can be obtained by mixing, casting and evaporating the aqueous polymer solution and the aqueous suspension. A first alternative consists in using an aqueous dispersed polymer, i.e. latex [6]. After mixing and casting the two aqueous suspensions, a 
solid nanocomposite film can be obtained by water evaporation and particles coalescence. A second alternative consists in using non aqueous systems. It means that the nanoparticles should be dispersed in an adequate, with respect to the polymeric matrix, organic medium. For instance it is possible to coat the surface of nanoparticles with a surfactant [7]. The chemical modification of nanoparticles surface is another way to disperse these nanoparticles in organic solvents. It generally involves reactive hydroxyl groups from the surface [8]. Recently, it was also shown that cellulose whiskers could be dispersed in dimethylformamide, dimethyl sulfoxide or $\mathrm{N}$-methyl pyrrolidine without additives or any surface modifications [9]. A solvent exchange procedure can also be used. Other possible processing techniques of nanocomposites are filtration of the suspension to obtain a film and then immersion in a polymer solution [10].

Although being widely used for the processing of thermoplastic polymers and composites reinforced with short fibers, very few studies have been reported concerning the processing of cellulose nanocrystals reinforced nanocomposites by melt extrusion methods. An attempt to prepare nanocomposites based on cellulose whiskers obtained from microcrystalline cellulose (MCC) and poly lactic acid (PLA) by melt extrusion technique was recently reported [11]. The suspension of nanocrystals was pumped into the polymer melt during the extrusion process. An attempt to use polyvinyl alcohol (PVA) as a compatibilizer to promote the dispersion of cellulose whiskers within the PLA matrix was reported [12].

In the present work, cellulose whiskers were functionalized by an esterification reaction with organic acid chloride aliphatic chains of different sizes. The objective of this surface chemical treatment was to enhance the non polar nature of the grafted nanocrystals and improve their dispersibility in a hydrophobic polymeric matrix. Low density polyethylene (LDPE) was chosen as the matrix and the properties of ensuing extruded nanocomposites were analyzed as a function of the whiskers content and length of the grafted chains.

\section{Materials and methods}

\subsection{Materials}

Ramie fibers were obtained from Stucken Melchers $\mathrm{GmbH} \&$ Co. (Germany). The low density polyethylene (LDPE) used for the study was PB-608 obtained from Braskem. It has a room temperature density of 0.915 g.cm ${ }^{-3}$ and Melt Flow Index $\left(190^{\circ} \mathrm{C} / 2.16 \mathrm{~kg}\right)$ of $30 \mathrm{~g} / 10$ min. Sulfuric acid (95\%), triehylamine (TEA, 99.5\%), toluene (anhydrous, 99.8\%), acetone (99\%), hexanoyl chloride, lauroyl chloride and stearoyl chloride (99\%) were all obtained from Sigma-Aldrich. The chemical structures of the various chemical grafting agents as well as their typical dimensions compared to cellulose whiskers are reported in Figure 1.

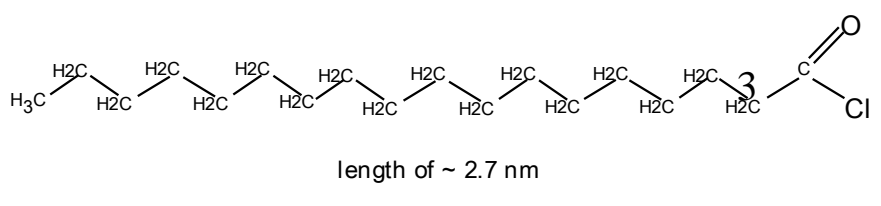

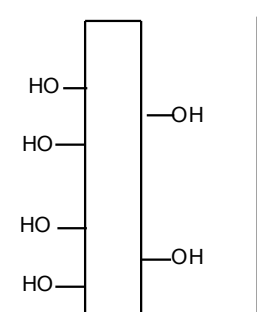


Figure 1: Chemical structures of the various chemical grafting agents and their typical dimensions compared to cellulose whiskers.

\subsection{Preparation of cellulose nanocrystals or whiskers}

Ramie fibers were first cut in small pieces and treated with a $2 \% \mathrm{NaOH}$ solution at $80^{\circ} \mathrm{C}$ for 2 hours to remove residual additives. Then, the ramie fibers were submitted to an acid hydrolysis treatment with a $65 \mathrm{wt} \% \mathrm{H}_{2} \mathrm{SO}_{4}$ solution at $55^{\circ} \mathrm{C}$ for $30 \mathrm{~min}$ under continuous stirring. The suspension was washed with water until neutrality and dialyzed with deionized water. The obtained suspension was homogenized with an Ultra Turrax T25 homogenizer at $13,500 \mathrm{rpm}(2-5 \mathrm{~min})$ and then filtered in sintered glass $\mathrm{N} N 1$.

\subsection{Surface chemical modification of cellulose whiskers}

The surface chemical modification of the cellulose whiskers was performed in a roundbottomed reaction flask under reflux (4 hours) and under constant mechanical stirring in toluene medium. The toluene suspension was obtained by a solvent exchange procedure and centrifugation procedure (water to acetone, and then acetone to toluene, both four times). Ramie nanocrystals $(2 \mathrm{~g})$ were mixed with triethylamine $(5 \mathrm{~mL})$ and organic acid chloride (5.2 $\mathrm{mL}$ for hexanoyl, $8.8 \mathrm{ml}$ for lauroyl or $12.5 \mathrm{~mL}$ for stearoyl chloride). Triethylamine was utilized to catalyze the reaction and as a complexing agent for $\mathrm{HCl}$ formed during the reaction [13]. The modified nanocrystals were submitted to a soxhlet extraction with acetone for 24 hours. The ramie cellulose whiskers modified with hexanoyl chloride, lauroyl chloride and stearoyl chloride will be denoted WRC6, WRC12 and WRC18, respectively, whereas unmodified whiskers will be later on denoted WRU. The scheme of the reaction was published elsewhere [13].

\subsection{Processing of nanocomposite materials}


Nanocomposite materials were prepared by mixing LDPE and either unmodified or chemically modified ramie cellulose whiskers (whiskers content ranging from 0 to $15 \mathrm{wt} \%$ ) using a twin-screw DSM Micro 15 compounder. The filler was used in the dry state after water or toluene evaporation for unmodified and functionalized cellulose whiskers, respectively. The components were introduced in the mixing chamber and allowed to melt at $160^{\circ} \mathrm{C}$. The mixing speed was set at $60 \mathrm{rpm}$ for $10 \mathrm{~min}$. The ensuing mixture was hot-pressed using a laboratory press (St-Eloi Mécanique) at $160^{\circ} \mathrm{C}$ under a force of 10 ton. This temperature was low enough, even under shear during the extrusion process, to avoid cellulose degradation.

\subsection{Characterization}

2.5.1. FTIR spectroscopy: FTIR spectrograms were obtained on a Perkin-Elmer Paragon 1000 FTIR spectrometer. Both unmodified and chemically modified ramie cellulose whiskers were analyzed as $\mathrm{KBr}$ pellets (1:100). The spectra were obtained with a resolution of $1 \mathrm{~cm}^{-1}$, averaging over 16 scans.

2.5.2. XPS spectroscopy: X-ray photoelectron spectrograms were performed using a XR3E2 (Vacuum Generator, UK) instrument equipped with monochromated $\mathrm{Mg} \mathrm{K} \alpha \mathrm{X}$-ray source $(1253.6 \mathrm{eV})$ and operated at $15 \mathrm{kV}$ under a current of $20 \mathrm{~mA}$.

2.5.3. Elemental analysis: Duplicate elemental analysis was carried out at the Laboratoire Central d'Analyses de Vernaison, France (CNRS). This technique is based on atomic absorption of the investigated elements. The carbon, nitrogen, and oxygen contents of cellulose nanocrystals were measured independently.

2.5.4. X-ray diffraction: X-ray diffraction data were recorded in reflection mode for dry whiskers powder and LDPE based nanocomposite films at room temperature with a Siemens D500 diffractometer equipped with a CuK $\alpha$ anode $(\lambda=0.15406 \mathrm{~nm})$.

2.5.5. Transmission electron microscopy (TEM): Drops of cellulose whiskers suspension were deposited on carbon-coated electron microscope grids and negatively stained with uranyl acetate solution. A Philips transmission electron microscope at an acceleration voltage of 80 $\mathrm{kV}$ was used.

2.5.6. Contact Angle measurements: Contact angle measurements were performed at room temperature with an OCA20 (DataPhysiscs Intruments). Three different liquids (water, $N, N$ dimethyl formamide and diiodomethane) with different dispersive and polar surface tensions were used to determine the surface energy of both unmodified and modified ramie whiskers, using the approach proposed by Owens and Wendt [14].

2.5.7. Thermogravimetric Analysis (TGA): TGA was carried out with a Shimadzu TA-50 instrument at $20^{\circ} \mathrm{C} \cdot \mathrm{min}^{-1}$ in a nitrogen atmosphere, from 25 to $900^{\circ} \mathrm{C}$. Samples were obtained by cutting the plates in circular form and weighed. 
2.5.8. Differential Scanning Calorimetry (DSC): DSC was performed using a DSC Q100 differential scanning calorimeter from TA Instruments. Samples were heated from -100 to $150^{\circ} \mathrm{C}$ at a heating rate of $10^{\circ} \mathrm{C} \mathrm{min}^{-1}$ under $\mathrm{N}_{2}$ atmosphere.

2.5.9. Dynamic mechanical analysis (DMA): DMA measurements were performed with a RSA3 (TA Instruments) working in the tensile mode. The sample dimensions were $10 \times 5 \mathrm{x}$ $0.2 \mathrm{~mm}^{3}$ and tests were performed under isochronal conditions at $1 \mathrm{~Hz}$ and the temperature was varied between -100 and $110^{\circ} \mathrm{C}$ at a heating rate of $5^{\circ} \mathrm{C} \min ^{-1}$.

2.5.10. Tensile tests: The non linear mechanical properties of the composites were carried out with a RSA3 (TA Instruments) with a load cell of 100N. Experiments were performed at room temperature with a crosshead speed of $10 \mathrm{~mm} \mathrm{~min}^{-1}$. The sample dimensions were $10 \mathrm{x}$ $5 \times 0.2 \mathrm{~mm}^{3}$ and the results were averaged over five measurements.

\section{Results and discussion}

\subsection{Characterization of ramie cellulose whiskers}

3.1.1. Morphological analysis. Acid hydrolysis of native ramie cellulose fibers leads to aqueous suspensions of elongated nanocrystals with high aspect ratio. Figure 2A shows an electron micrograph of ramie cellulose whiskers. The length and diameter of these nanocrystals were determined by using digital image analysis (ImageJ). Both the obtained histograms and cumulative curves are shown in Figure 2B. The geometric average length and diameter were around $134 \mathrm{~nm} \pm 59 \mathrm{~nm}$ and $10.8 \mathrm{~nm} \pm 4.5 \mathrm{~nm}$, respectively, giving rise to an aspect ratio around 12. A minimum of 228 and 70 measurements were used to determine the length and the diameter, respectively, of ramie whiskers. It is worth noting that more than $50 \%$ of the nanoparticles have a length lower than $100 \mathrm{~nm}$.

Regarding their arrangement in solution, it is noteworthy that because of electrostatic repulsions between surface-grafted sulfate ester groups resulting from the sulfuric acid hydrolysis, the cellulose whiskers repel each other and then do not flocculate in water. The overall concentration of these sulfate moieties is related to the sulfur ratio, which was determined by elementary analysis to be $0.57 \%$ of dry matter [15]. According to these sulfur ratios and to the average geometry of cellulose nanocrystals, the average surface charge of rods was estimated to $0.60 \mathrm{e.nm}^{-2}$ (considering rod-like nanoparticles with an average diameter of 6-8 $\mathrm{nm}$ and a length of about 150-250 nm).

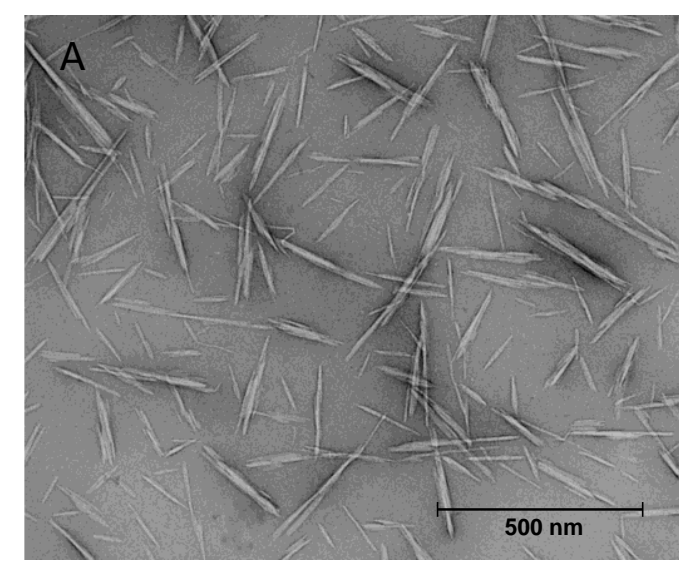


Length : $134 \mathrm{~nm} \pm 59 \mathrm{~nm}$

(228 measurements)

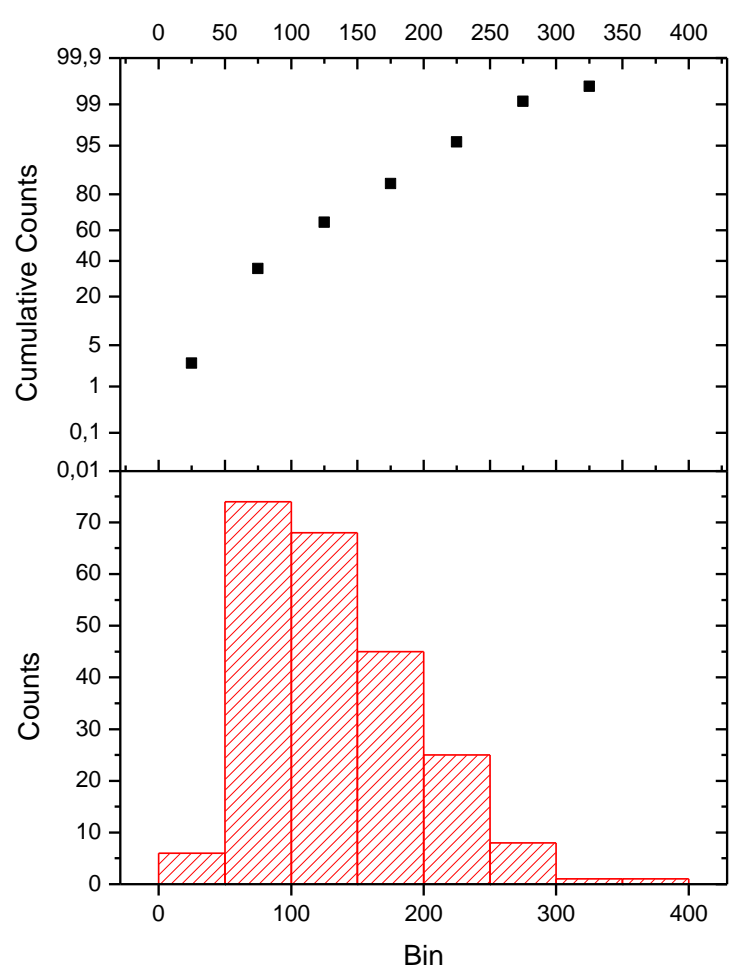

Diameter: $10.8 \mathrm{~nm} \pm 4.5 \mathrm{~nm}$

(70 measurements)

B

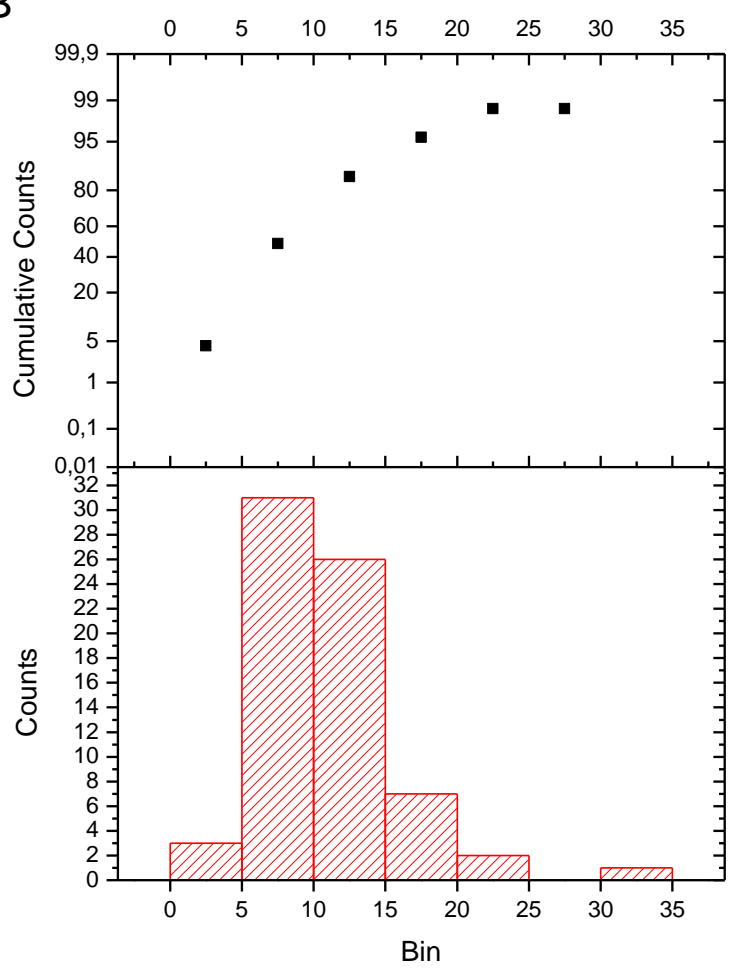

Figure 2: Transmission electron micrograph (A) and length and diameter histograms and cumulative curves of ramie cellulose whiskers (B).

3.1.2. FTIR investigation. The FTIR spectra recorded for both unmodified and chemically modified ramie whiskers are shown in Figure 3A. Compared to unmodified whiskers, the spectra corresponding to modified cellulose nanoparticles display an extra peak at $1737 \mathrm{~cm}^{-1}$, attributed to carbonyl groups. The signals at 2953, 2919 and $2850 \mathrm{~cm}^{-1}$ are ascribed to the presence of grafted alkane chains. The concomitant decrease of the magnitude of the broad band around $3300 \mathrm{~cm}^{-1}$ for modified whiskers compared to unmodified is attributed to the partial disappearance of $\mathrm{OH}$ groups, confirming the success of the grafting reaction with organic acid chlorides.

3.1.3. XPS analysis. Figure 3B shows the XPS spectra for both unmodified and chemically grafted ramie whiskers. The signals observed around binding energies of 531 and $287 \mathrm{eV}$ correspond to the $1 \mathrm{~s}$ orbital electron of oxygen and carbon, respectively. The elemental surface composition (\%) and the oxygen-to-carbon ratios of the different samples are summarized in Table 1. It decreases from 0.51 for the unmodified cellulose whiskers to 0.36 for the sample grafted with $\mathrm{C} 18$ chains. It is also observed that the $\mathrm{O} / \mathrm{C}$ ratio decreases with 
increasing the length of the aliphatic grafted chains typical of organic acid chloride aliphatic chains. Figure 4 presents the deconvolution of the $\mathrm{C} 1 \mathrm{~s}$ peak that is composed of the $\mathrm{C} 1$ peak corresponding to $\mathrm{C}-\mathrm{C} / \mathrm{C}-\mathrm{H}$ linkages, $\mathrm{C} 2$ peak corresponding to $\mathrm{C}-\mathrm{O}$ of alcohols and ethers, $\mathrm{C} 3$ peak corresponding to the $\mathrm{O}-\mathrm{C}-\mathrm{O}$ and $\mathrm{C}=\mathrm{O}$ from the acetal moieties and $\mathrm{C} 4$ peak, corresponding to $\mathrm{O}-\mathrm{C}=\mathrm{O}$ and representing the ester carbon contribution. The peak corresponding to carbon was deconvoluted for each sample using curve fitting software (Spectrum NT).
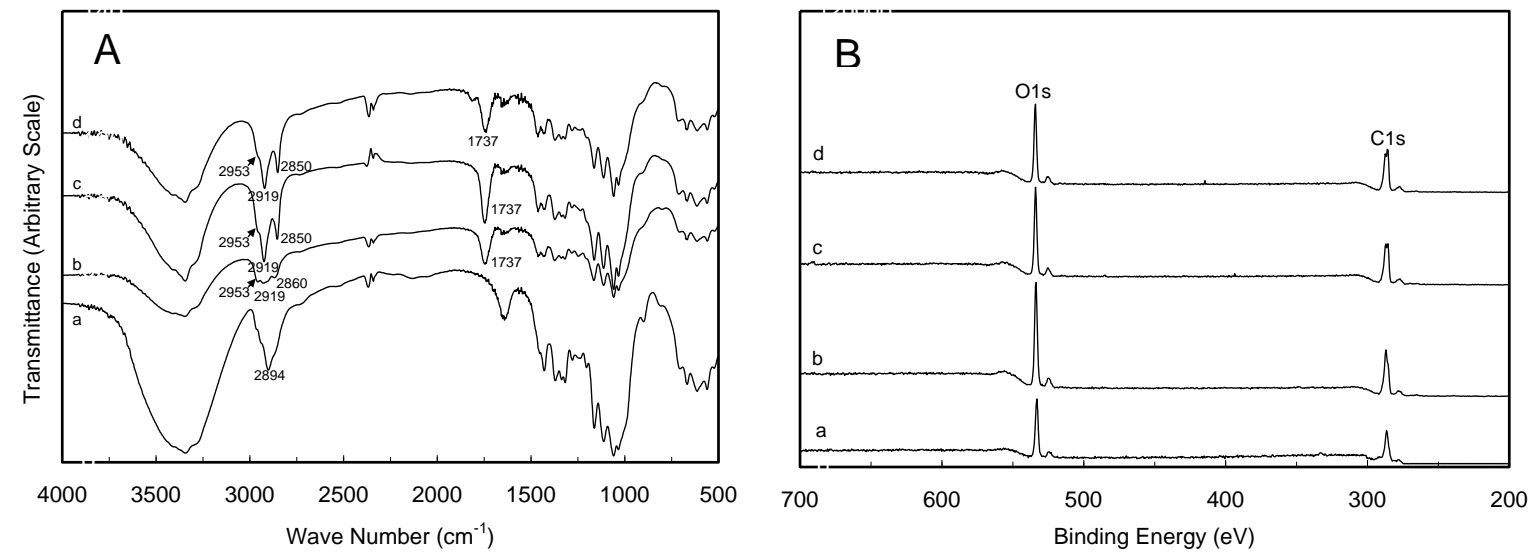

Figure 3: FTIR spectra (KBr pellets) (A) and general XPS spectra (B) of unmodified ramie cellulose whiskers (a) and surface modified with hexanoyl chloride (b), lauroyl chloride (c) and stearoyl chloride (d), after soxhlet extraction with acetone.

Table 1: XPS analysis of ramie cellulose whiskers after and before surface chemical modification with hexanoyl, lauroyl and stearoyl chloride.

\begin{tabular}{ccccccc}
\hline \multicolumn{7}{c}{ Binding energy $(\mathrm{eV})$} \\
\hline \multirow{2}{*}{ Sample } & $\mathrm{O} / \mathrm{C}$ & $\begin{array}{c}\mathrm{C} 1 \\
285 \pm 0.1 \\
\mathrm{C}-\mathrm{C} / \mathrm{C}-\mathrm{H}\end{array}$ & $\begin{array}{c}\mathrm{C} 2 \\
\mathrm{C}-\mathrm{O}\end{array}$ & $\begin{array}{c}\mathrm{C} 3 \\
\mathrm{O}-\mathrm{C}-\mathrm{O} / \mathrm{C}=\mathrm{O}\end{array}$ & $\begin{array}{c}\mathrm{C} 4 \\
\mathrm{O}-\mathrm{C}=\mathrm{O}\end{array}$ & $\theta_{\mathrm{SC}}(\%)$ \\
& & & $287.7 \pm 0.1$ & \\
\hline WRU & 0.51 & 27.4 & 52.8 & 17.2 & 2.6 & ---- \\
WRC6 & 0.53 & 34.5 & 46.4 & 14.6 & 4.5 & 7.1 \\
WRC12 & 0.41 & 45.0 & 38.4 & 12.6 & 3.9 & 17.6 \\
WRC18 & 0.36 & 50.4 & 37.8 & 9.8 & 1.9 & 23.0 \\
\hline
\end{tabular}



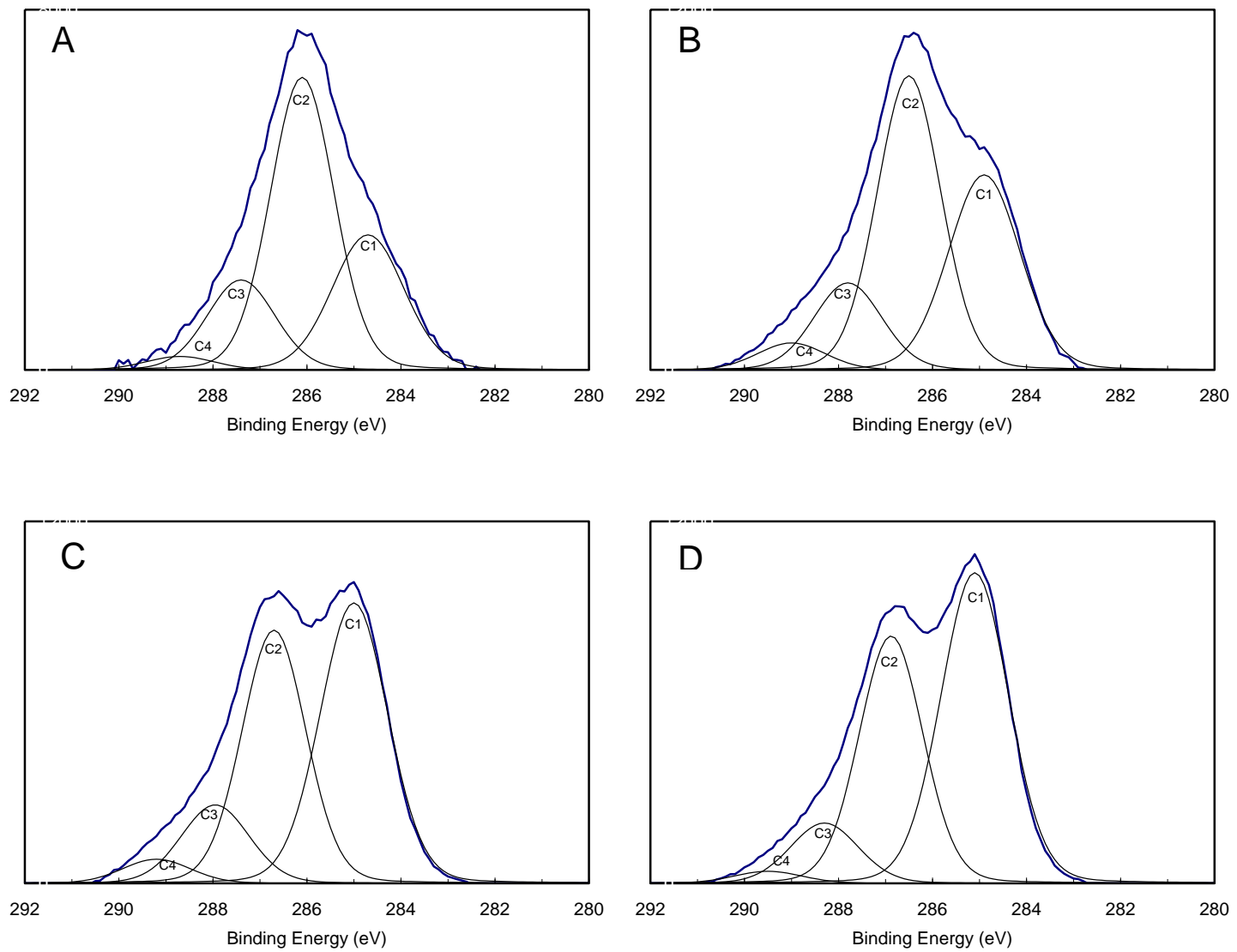

Figure 4: Decomposition of $\mathrm{C} 1 \mathrm{~s}$ signal into its constituent contributions for unmodified ramie cellulose whiskers (A) and modified with hexanoyl chloride (B), lauroyl chloride (C) and stearoyl chloride (D).

Considerable changes can be observed in the intensities of the $\mathrm{C} 1$ peak, referring to aliphatic chains $(\mathrm{C}-\mathrm{H})$, for modified samples when compared to the pristine sample. This behavior can be attributed to the chain grafting at the whisker surface and provides additional proof of successful grafting of aliphatic chains to cellulose. Indeed, XPS is a powerful tool to investigate chemical changes resulting from surface modification since the investigated thickness is of the order of about $6 \mathrm{~nm}$. Values of the $\mathrm{C} 1, \mathrm{C} 2, \mathrm{C} 3$ and $\mathrm{C} 4$ contributions are reported in Table 1 . The surface coverage by large alkane chain $\left(\theta_{\mathrm{SC}}\right)$ is confirmed, as estimated by the $\mathrm{C} 1$ relative area according to equation $1[16]$.

$$
\theta_{\mathrm{SC}}=\mathrm{A}_{\mathrm{C} 1 \mathrm{~m}}-\mathrm{A}_{\mathrm{C} 1 \mathrm{um}}
$$

where, $\mathrm{A}_{\mathrm{Clm}}$ and $\mathrm{A}_{\mathrm{Cl} \text { um }}$ are the areas of the modified and unmodified samples, respectively. The $\theta_{\mathrm{SC}}$ values are reported in Table 1 and its evolution as a function of the number of carbons of the grafted chain is shown in Figure 5. The increase of the surface coverage gives a clear evidence of the occurrence of the expected surface chemical modification of the ramie cellulose whiskers. 


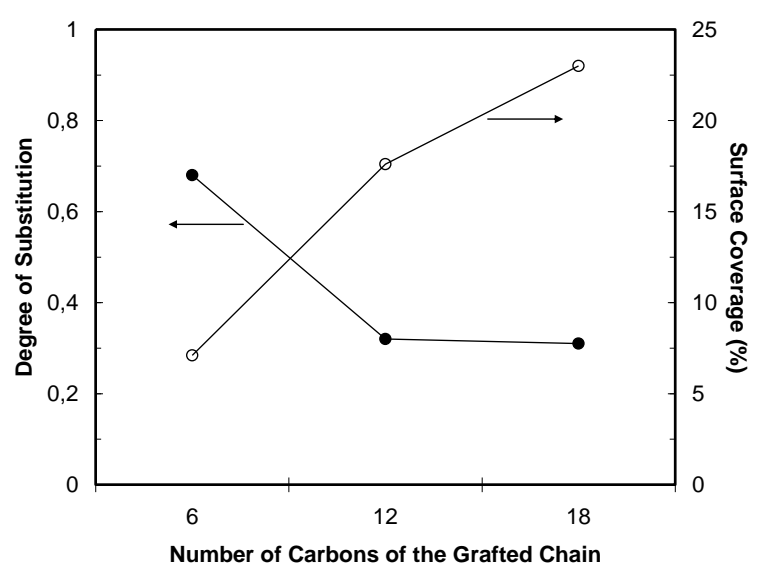

Figure 5: Evolution of the surface coverage area and degree of substitution (DS) as a function of the length of grafted chains. The lines serve to guide the eyes.

3.1.4. Elemental analysis. The elemental analyses of both unmodified and modified samples are given in Table 2. The degree of substitution (DS) of the modified ramie whiskers was determined from the data obtained by elemental analysis [17]. The DS values obtained from this technique are reported in Table 2 and its evolution as a function of the number of carbons of the grafted chain is shown in Figure 5. A decrease of DS is observed when the number of carbon atoms of the chloride acid increases and it seems to stabilize for highest chain lengths. This behavior was also observed after esterification of lignocellulosic materials [18].

Table 2: Elemental analyses of ramie cellulose nanocrystals before and after chemical modification with hexanoyl, lauroyl and stearoyl chloride.

\begin{tabular}{ccccc}
\hline Sample & $\mathrm{C}(\%)$ & $\mathrm{H}(\%)$ & $\mathrm{O}(\%)$ & $\mathrm{DS}$ \\
\hline WRU & 43.08 & 6.41 & 50.51 & ---- \\
WRC6 & 52.90 & 7.67 & 39.43 & 0.68 \\
WRC12 & 53.57 & 8.12 & 38.31 & 0.32 \\
WRC18 & 56.90 & 8.88 & 34.22 & 0.31 \\
\hline
\end{tabular}

3.1.5. X-ray diffraction. Natural lignocellulosic fibers are known to display $\mathrm{X}$ ray diffraction (XRD) patterns typical of cellulose type I, with the main diffraction signals at $2 \theta$ values of $15^{\circ}, 16^{\circ}, 22,5^{\circ}$ and $34^{\circ}$, attributed to the diffraction planes 101, 10 $\overline{1}, 002$ and 040 , respectively. Figure $6 \mathrm{~A}$ shows the $\mathrm{XRD}$ patterns obtained for the modified ramie cellulose whiskers samples as well as the one corresponding to the pristine sample. Even after chemical modification, the cellulosic nanoparticles remain semicrystalline and display the same XRD 
patterns leading to the conclusion that the initial crystallinity was retained. Then, the surface chemical modification did not alter the crystallinity of cellulose nanocrystals. Upon chemical modification, a new ill-defined peak, located by an arrow, appears around $21^{\circ}$ that is attributed to the presence of grafted aliphatic chains. In a previous work [19], a similar but much more defined peak was observed for polycaprolactone-grafted starch nanocrystals. This peak was much less defined for PCL-grafted cellulose whiskers because a strong diffraction peak due to cellulose I occurred in the same diffraction angle range.

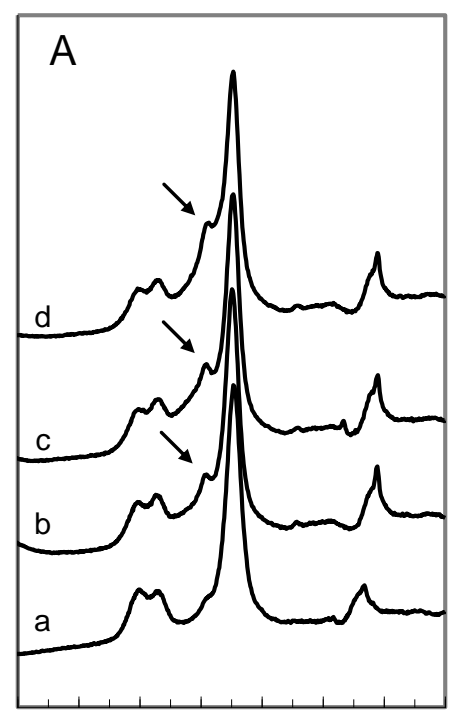

$\begin{array}{llllllll}5 & 10 & 15 & 20 & 25 & 30 & 35 & 40\end{array}$ $2 \theta\left({ }^{\circ}\right)$

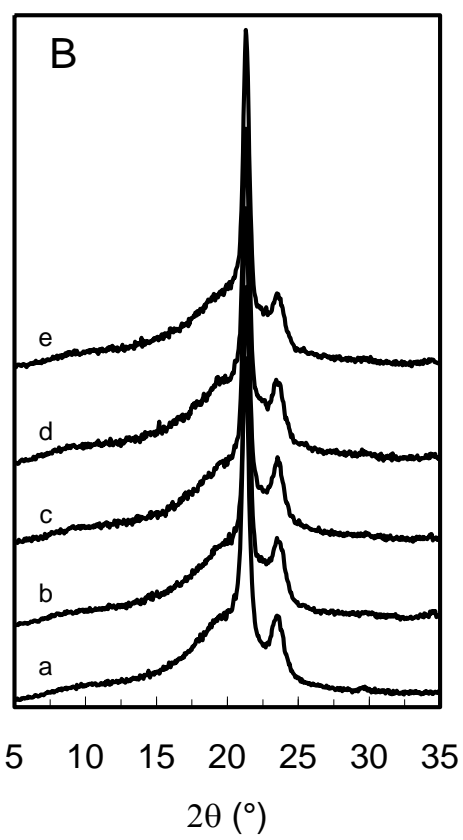

$2 \theta\left(^{\circ}\right)$

Figure 6: Wide-angle $\mathrm{X}$-ray diffraction patterns for ramie cellulose whiskers (A): unmodified (a) and modified with hexanoyl chloride (b), lauroyl chloride (c) and stearoyl chloride (d), and nanocomposite films (B): neat LDPE matrix (a) and related nanocomposite films reinforced with $10 \mathrm{wt} \%$ ramie cellulose whiskers: unmodified (b) and modified with hexanoyl chloride (c), lauroyl chloride (d) and stearoyl chloride (e).

3.1.6. Contact angle measurements. Table 3 shows the values of contact angle measured for ramie cellulose whiskers at longer times, i.e. when the equilibrium was reached. The values of the surface energy as well as the polar and dispersive contributions are also reported. The dynamic behavior of the contact angle for a drop of distilled water onto the surface of the materials is shown in Figure 7. The untreated cellulose surface gave the lowest initial contact angle value, which is an expected finding since the surface of cellulose nanoparticles contains $\mathrm{OH}$-rich macromolecules, the most capable to establish hydrogen bonds with water. The initial contact angle is two times higher for grafted substrates, indicating that chemical treatments induced dramatic changes in surface polarity of cellulose nanocrystals. The contact angle value increases as the length of the grafted chain increases. Moreover, whereas the contact angle value decreases with time for WRU and WRC6 substrates, it remains almost 
constant for WRC12 and WRC18. Then, contact angles measurements give a clear evidence of the change in hydrophobicity of the modified samples compared to the unmodified sample. These results were confirmed by the decrease of the polar contribution to the surface energy (Table 3).

The initial contact angle values, corresponding to times $\sim 0$, for WRU and WRC6 are 45 and 87, respectively. After few seconds, it remains almost constant with values of 35 and 72 . It was checked that the decrease of the contact angle value was due a spreading of the drop rather than a penetration by capillarity because of the porous character of the pellets prepared for these measurements. It was checked that the diameter of the drop increased and that the volume of the drop remained constant during time.

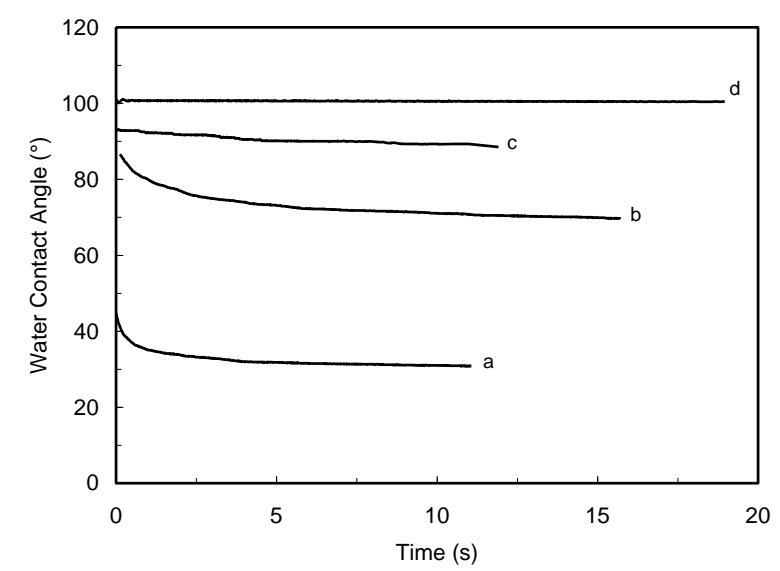

Figure 7: Water contact angle with time for unmodified ramie cellulose whiskers (a) and modified with hexanoyl chloride (b), lauroyl chloride (c) and stearoyl chloride (d).

Table 3: Contact angles and surface tension contributions of the ramie cellulose whiskers before and after modification with hexanoyl, lauroyl and stearoyl chloride.

Contact Angle

\begin{tabular}{ccccccc}
\hline Sample & Water & Formamide & Diiodomethane & $\gamma_{\mathrm{s}}^{\mathrm{p}}\left(\mathrm{mJ} / \mathrm{m}^{2}\right)$ & $\gamma_{\mathrm{s}}{ }^{\mathrm{d}}\left(\mathrm{mJ} / \mathrm{m}^{2}\right)$ & $\gamma_{\mathrm{s}}^{\mathrm{T}}\left(\mathrm{mJ} / \mathrm{m}^{2}\right)$ \\
\hline WRU & 35 & 19 & 40 & 35.2 & 25.3 & 60.5 \\
WRC6 & 72 & 40 & 61 & 12.5 & 25.6 & 38.1 \\
WRC12 & 90 & 55 & 61 & 2.9 & 30.0 & 32.9 \\
WRC18 & 101 & 75 & 56 & 0.2 & 31.3 & 31.5 \\
\hline
\end{tabular}

3.1.7. Thermal analysis. The thermal degradation behavior of the cellulose nanocrystals was investigated from TGA measurements. Results are reported in Figure 8. The unmodified sample displays a weight loss from room temperature to $130^{\circ} \mathrm{C}$. It is ascribed to the presence of water. This effect is decreased for the sample modified with C6 and absent for other specimens, i.e. nanocrystals modified with $\mathrm{C} 12$ and C18. It is obviously ascribed to a lower accessibility of surface $\mathrm{OH}$ groups after the grafting reaction. Then, a higher weight loss is observed in the range $250-350^{\circ} \mathrm{C}$. The thermal decomposition temperatures, associated with a 
$2 \%$ weight loss and maximum of derived signal, were determined and results are collected in Table 4. The degradation temperature is lower for modified nanoparticles compared to the pristine cellulose whiskers and it decreases as the length of the grafted chains increases as already reported for another system [20].
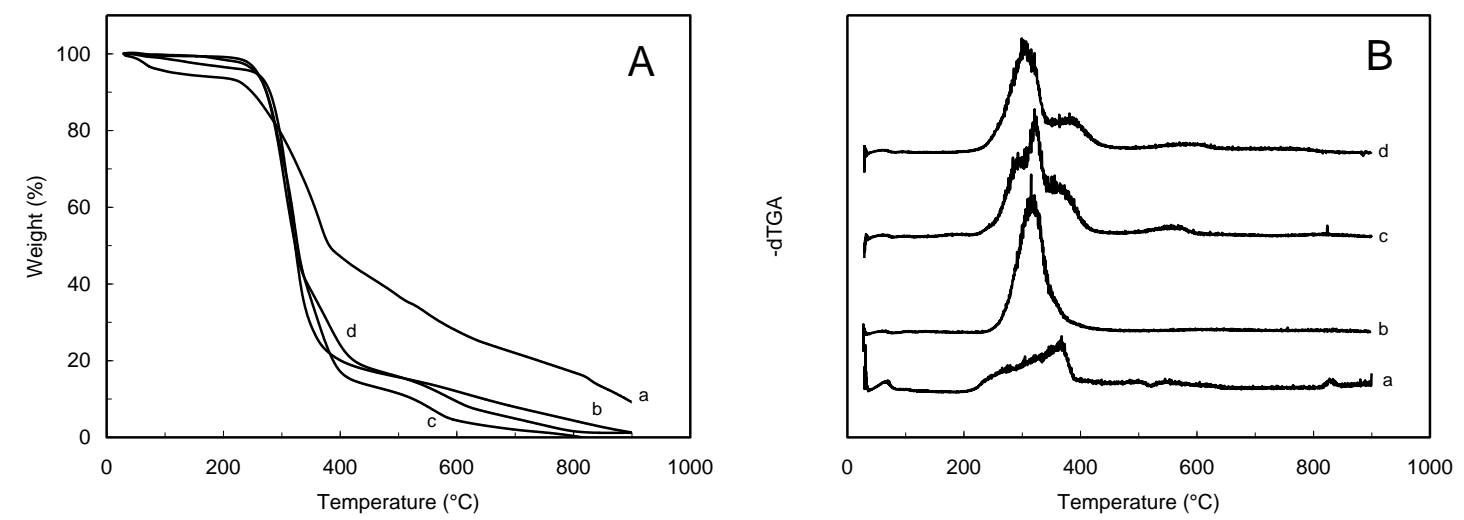

Figure 8: TGA curves (A) and dTGA (B) for unmodified ramie cellulose whiskers (a) and modified with hexanoyl chloride (b), lauroyl chloride (c) and stearoyl chloride

Table 4: Degradation data obtained from TGA measurements for unmodified and modified ramie whiskers: $\mathrm{T}_{\text {onset }}$ corresponds to the beginning of the degradation process, and $\mathrm{Td}_{1}$ and $\mathrm{Td}_{2}$ to the thermal decomposition temperature associated with a $2 \%$ weight loss and maximum of derived signal, respectively. Measurements were performed under nitrogen flow at $20^{\circ} \mathrm{C} \cdot \mathrm{min}^{-1}$.

\begin{tabular}{cccc}
\hline Samples & $\begin{array}{c}\mathrm{T}_{\text {onset }} \\
(\leq 0.5 \% \text { weight }\end{array}$ & $\begin{array}{c}\text { Td }\left({ }^{\circ} \mathrm{C}\right) \\
(\text { lass })\end{array}$ & $\begin{array}{c}\text { Td maximum by } \\
\text { dTGA }\left({ }^{\circ} \mathrm{C}\right)\end{array}$ \\
\hline WRU & 210 & 240 & 368 \\
WRC6 & 242 & 267 & 320 \\
WRC12 & 200 & 236 & 321 \\
WRC18 & 215 & 248 & 305 \\
\hline
\end{tabular}

The DSC thermograms obtained for both unmodified and chemically modified nanoparticles are shown in Figure 9. Panel A corresponds to the first temperature scan, whereas panel B corresponds to the second temperature scan, the samples being quenched between each temperature scan. An endothermic peak is observed around $110^{\circ} \mathrm{C}$ for the pristine sample during the first temperature scan. This peak is not observed during the second temperature scan and was ascribed to the vaporization of water. For modified samples, it is not observed because of the increased hydrophobicity of the nanoparticles. However, the thermogram recorded for cellulose whiskers modified with $\mathrm{C} 18$ during the first temperature scan (Figure 9A-curve d) shows a double endothermic peak between 25 and $80^{\circ} \mathrm{C}$. It is ascribed to the melting of covalently linked chains at the cellulose nanocrystal surface that were sufficiently long to crystallize. The formation of a crystalline brushlike structure of stearate moieties grafted to starch nanoparticle surface was reported elsewhere [13]. Even after quenching, a 
melting endotherm is observed for the WRC18 sample (Figure 9B) showing that the kinetics of crystallization of grafted chains is fast. However, the global aspect of this melting endotherm is different from the one recorded during the initial temperature scan.
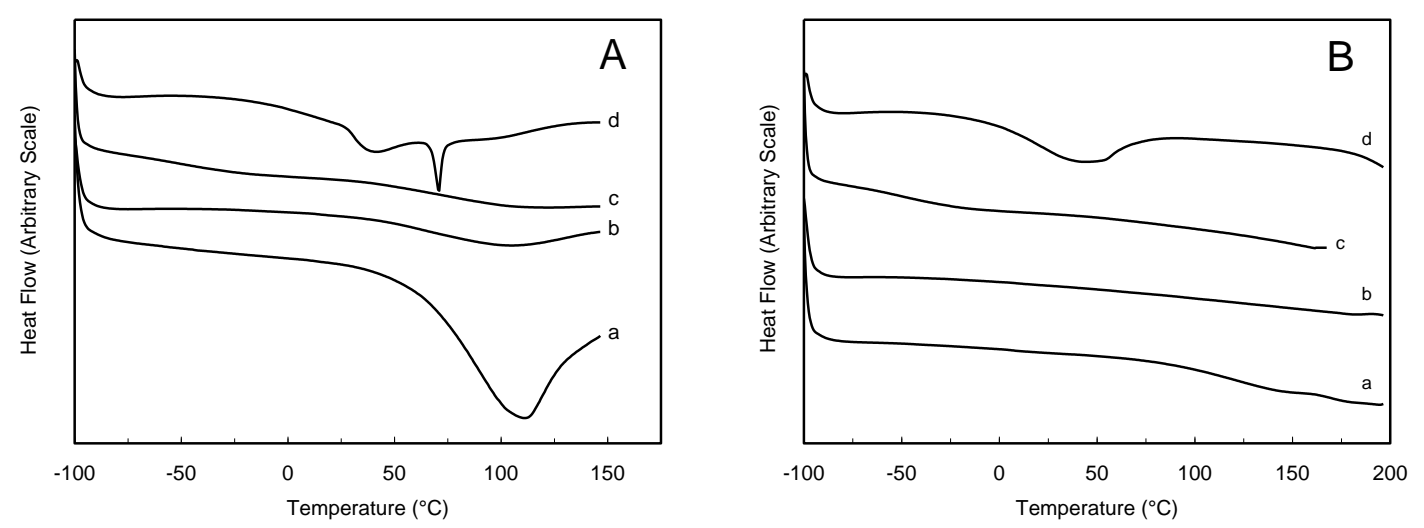

Figure 9: DSC traces for unmodified ramie cellulose whiskers (a) and modified with hexanoyl chloride (b), lauroyl chloride (c) and stearoyl chloride (d). First temperature scan (A) and second temperature scan recorded after the first temperature scan and subsequent quenching (B). Traces have been shifted vertically.

\subsection{Characterization of ramie cellulose whiskers reinforced LDPE nanocomposite films}

3.2.1. Morphological analysis. Figure 10 shows photographs of the unfilled LDPE film and ramie cellulose whiskers based nanocomposite films reinforced with $10 \mathrm{wt} \%$ of WRU and WRC18. The neat PE is obviously translucent as any low thickness polymeric film with a relatively low degree of crystallinity. When adding $10 \mathrm{wt} \%$ of cellulose whiskers, the film becomes dotted with black. These heterogeneities reveal the poor and inhomogeneous dispersion of the filler within the polymeric matrix. It is obviously ascribed to the highly hydrophobic nature of the matrix and highly hydrophilic character of the cellulose nanoparticles. When the cellulose whiskers were chemically modified with aliphatic chains, the occurrence of these aggregates progressively vanish and the appearance of the composite film reinforced with WRC18 becomes similar to the one of the unfilled film. In addition, it is worth noting that it seems that the cellulose whiskers are not orientated when extruded in the melt. Indeed, we observed that no difference was reported for extruded films reinforced with cellulose whiskers when testing the film in the flow or transverse direction. These results are reported in a forthcoming publication.

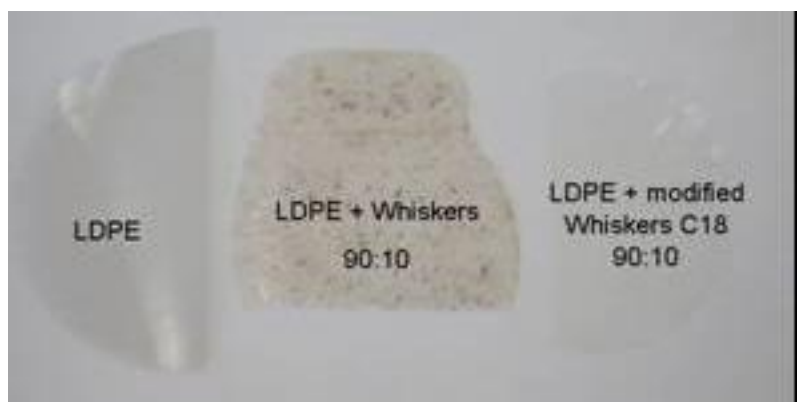


Figure 10: Photographs of the neat film and ramie cellulose whiskers based nanocomposite films reinforced with $10 \mathrm{wt} \%$ of WRU and WRC 18 .

3.2.2. Thermal analysis. The thermal characterization of ramie cellulose whiskers reinforced LDPE nanocomposite films was carried out using DSC. From the analysis of DSC traces, the melting temperature $\left(\mathrm{T}_{\mathrm{m}}\right)$, associated heat of fusion $\left(\Delta \mathrm{H}_{\mathrm{m}}\right)$ and degree of crystallinity $\left(\chi_{\mathrm{c}}\right)$ were obtained for the unfilled LDPE film, and nanocomposite materials reinforced with either unmodified or modified whiskers. The resulting experimental data are listed in Table 5. It is worth noting that for the calculation of the degree of crystallinity of the composite materials, the heat of fusion was normalized to the matrix content.

Table 5: Melting characteristics of LDPE-based nanocomposites reinforced with ramie cellulose whiskers obtained from DSC measurements: melting temperature $\left(T_{m}\right)$, enthalpy of fusion $\left(\Delta \mathrm{H}_{\mathrm{m}}\right)$ and degree of crystallinity $\left(\chi_{\mathrm{c}}\right)$.

\begin{tabular}{ccccc}
\hline Sample & Whisker Content $(\%)$ & $\mathrm{T}_{\mathrm{m}}\left({ }^{\mathrm{o}} \mathrm{C}\right)$ & $\Delta \mathrm{H}_{\mathrm{m}}\left(\mathrm{J} \cdot \mathrm{g}^{-1}\right)$ & $\chi_{\mathrm{c}}{ }^{\mathrm{a}}$ \\
\hline LDPE & 0 & 103 & 110.1 & 0.38 \\
\hline \multirow{2}{*}{ LDPE-WRU } & 3 & 104 & 114.0 & 0.41 \\
& 5 & 103 & 113.4 & 0.41 \\
& 10 & 103 & 127.2 & 0.49 \\
LDPE-WRC6 & 15 & 103 & 124.0 & 0.51 \\
& 3 & 104 & 110.2 & 0.39 \\
& 5 & 103 & 116.3 & 0.43 \\
LDPE-WRC12 & 10 & 104 & 124.3 & 0.48 \\
& 15 & 105 & 127.1 & 0.52 \\
\hline \multirow{3}{*}{ LDPE-WRC18 } & 3 & 103 & 112.4 & 0.40 \\
& 5 & 104 & 119.2 & 0.44 \\
& 10 & 103 & 130.9 & 0.51 \\
& 15 & 104 & 125.2 & 0.51 \\
\hline & 5 & 105 & 115.7 & 0.41 \\
& 10 & 103 & 122.0 & 0.45 \\
& 15 & 105 & 132.9 & 0.51 \\
\hline
\end{tabular}

${ }^{\mathrm{a}} \chi_{\mathrm{c}}=\Delta \mathrm{H}_{\mathrm{m}} / \mathrm{w} \Delta \mathrm{H}_{\mathrm{m}}$, where $\Delta \mathrm{H}_{\mathrm{m}}=290 \mathrm{~J} / \mathrm{g}$ (heat of fusion for $100 \%$ crystalline LDPE) and $\mathrm{w}$ is the weight fraction of polymeric matrix in the composite.

The melting point remains roughly constant between 103 and $105^{\circ} \mathrm{C}$ upon whiskers addition, regardless their modification state. It is an indication that the size of the crystallites is not affected by the filler. On the contrary, the degree of crystallinity of the LDPE matrix was found to increase with the whiskers content nearly independently of their nature (Figure 11), their surface being chemically modified or not. It seems that the cellulosic nanoparticles probably act as nucleating agents for the polymeric matrix. This nucleating effect is surprisingly not influenced by the grafting of aliphatic chains at the nanoparticle surface. Indeed, because it has been shown from DSC measurements that, at least for WRC18 
nanoparticles, grafted chains can crystallize at the surface of cellulose whiskers, one could expect a possible co-crystallization of covalently linked chains at the nanoparticles surface with those from the matrix. However, it is worth noting that for modified whiskers reinforced composites, the nanocrystal content refers to the weight fraction of grafted nanoparticles and that the effective whisker content is lower compared to unmodified. It means that experimental data for modified nanoparticles based composites in Figure 11 should be shifted horizontally towards the lower whiskers content.

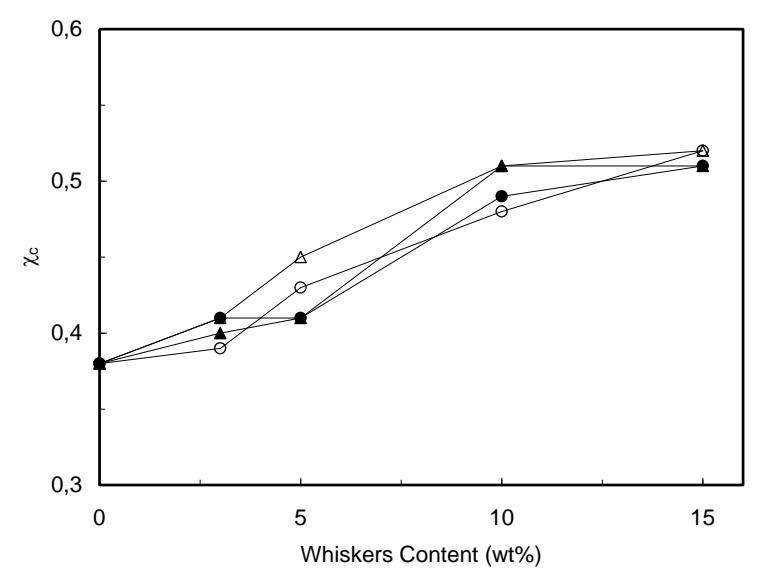

Figure 11: Evolution of the degree of crystallinity of the LDPE matrix as a function of the ramie cellulose whiskers content for nanocomposite films reinforced with unmodified $(\bullet)$ and modified with hexanoyl chloride $(\circ)$, lauroyl chloride $(\boldsymbol{\Delta})$ and stearoyl chloride $(\Delta)$ whiskers. The lines serve to guide the eyes.

The thermal degradation behavior of ramie cellulose whiskers reinforced LDPE nanocomposite films was investigated from TGA measurements. Results are reported in Table 6. The onset temperature of the weight loss process was systematically lower for composites than for the neat matrix. It is ascribed to the water content of the cellulosic filler. The degradation temperature associated to the maximum of the dTGA signal remains roughly constant regardless the cellulose whiskers content and its surface modification.

Table 6: Thermal degradation characteristics $\left(T_{\text {onset }}\right.$ : onset of the degradation, $T_{d}$ : thermal decomposition temperature associated with a $2 \%$ weight loss, and $\mathrm{Td}$ maximum : maximum of the derived signal) for LDPE-based nanocomposite films reinforced with ramie cellulose whiskers immersed in water.

\begin{tabular}{ccccc}
\hline Sample & $\begin{array}{c}\text { Whisker } \\
\text { Content }(\%)\end{array}$ & $\begin{array}{c}\mathrm{T}_{\text {onset }} \\
(\geq 0.5 \% \text { weight loss })\end{array}$ & $\begin{array}{c}\text { Td }\left({ }^{\circ} \mathrm{C}\right) \\
(\text { at } 2 \% \text { weight loss })\end{array}$ & $\begin{array}{c}\text { Td maximum } \\
\text { by dTGA ( } \mathrm{C})\end{array}$ \\
\hline LDPE & 0 & 321 & 354 & 482 \\
\hline \multirow{3}{*}{ LDPE-WRU } & 3 & 263 & 310 & 488 \\
& 5 & 255 & 297 & 487 \\
& 10 & 247 & 283 & 484 \\
LDPE-WRC6 & 15 & 239 & 268 & 485 \\
& 3 & 293 & 334 & 480 \\
& 10 & 280 & 306 & 485 \\
& 5 & 276 & 293 & 487
\end{tabular}




\begin{tabular}{ccccc} 
& 15 & 271 & 288 & 488 \\
\hline \multirow{3}{*}{ LDPE-WRC12 } & 3 & 297 & 333 & 490 \\
& 5 & 281 & 320 & 474 \\
& 10 & 286 & 310 & 487 \\
& 15 & 281 & 302 & 493 \\
\hline \multirow{2}{*}{ LDPE-WRC18 } & 3 & 297 & 335 & 491 \\
& 5 & 290 & 324 & 495 \\
& 10 & 275 & 301 & 489 \\
& 15 & 256 & 282 & 480 \\
\hline
\end{tabular}

3.2.3. X-ray diffraction. Figure 6B shows the X-ray diffraction patterns obtained for the neat LDPE matrix and related nanocomposites reinforced with $10 \mathrm{wt} \%$ cellulose whiskers. No alteration of the diffraction pattern of LDPE is observed upon ramie whiskers addition indicating that the crystallinity of LDPE is not affected upon whiskers addition. However, it is worth noting that there might be local changes in crystallinity that the diffractometer cannot spatially resolve.

3.2.4. Mechanical properties. Figure 12 shows the evolution of the logarithm of the storage tensile modulus as a function of temperature for ramie cellulose whiskers reinforced LDPE nanocomposites. Panels A, B, C and D correspond to filler contents of 3, 5, 10 and $15 \mathrm{wt} \%$, respectively. The behavior of the neat LDPE matrix has been added in each Figure for reference and modulus values have been normalized at low temperature. The modulus drop observed around $-40^{\circ} \mathrm{C}$ is ascribed to the anelastic manifestation of the glass transition of the polymeric matrix. The modulus drop corresponding to this relaxation is weak because LDPE is a semicrystalline polymer. Indeed, the rubbery modulus is known to depend on the degree of crystallinity of the material, the crystalline regions of LDPE acting as physical cross-links for the elastomer. In this physically cross-linked system, the crystalline regions would also act as filler particles due to their finite size, which would increase the modulus substantially. At higher temperatures, E' decreases continuously because of the progressive melting of LDPE. At the melting point of the polymeric matrix, the modulus drops irreversibly and the setup fails to measure it, due to irreversible chain flow.
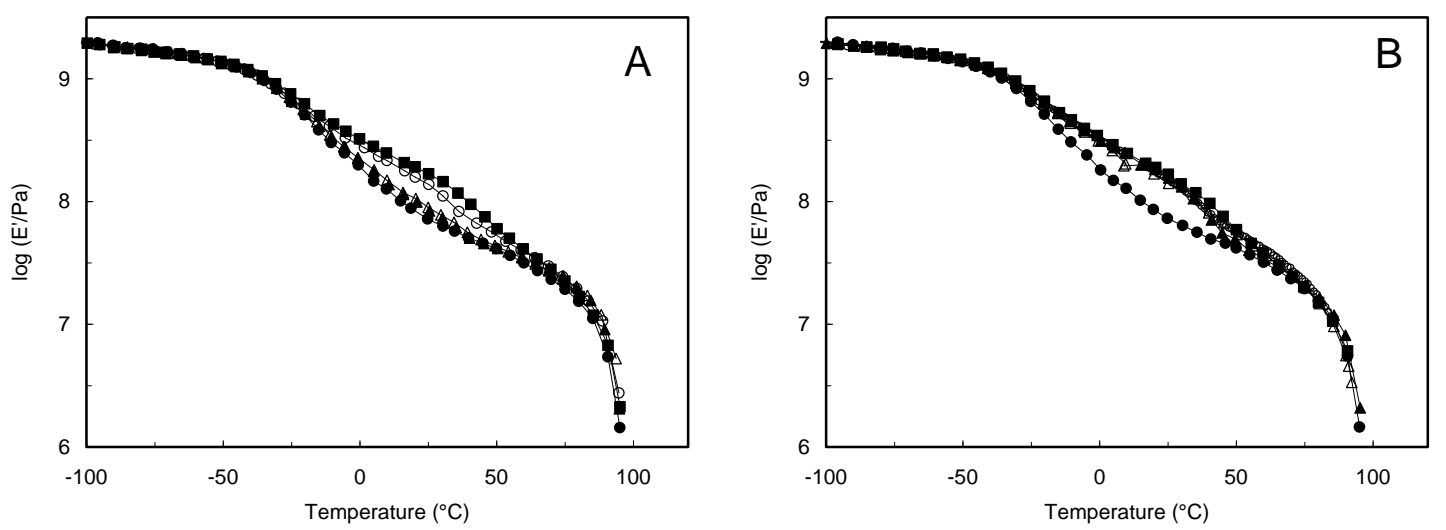

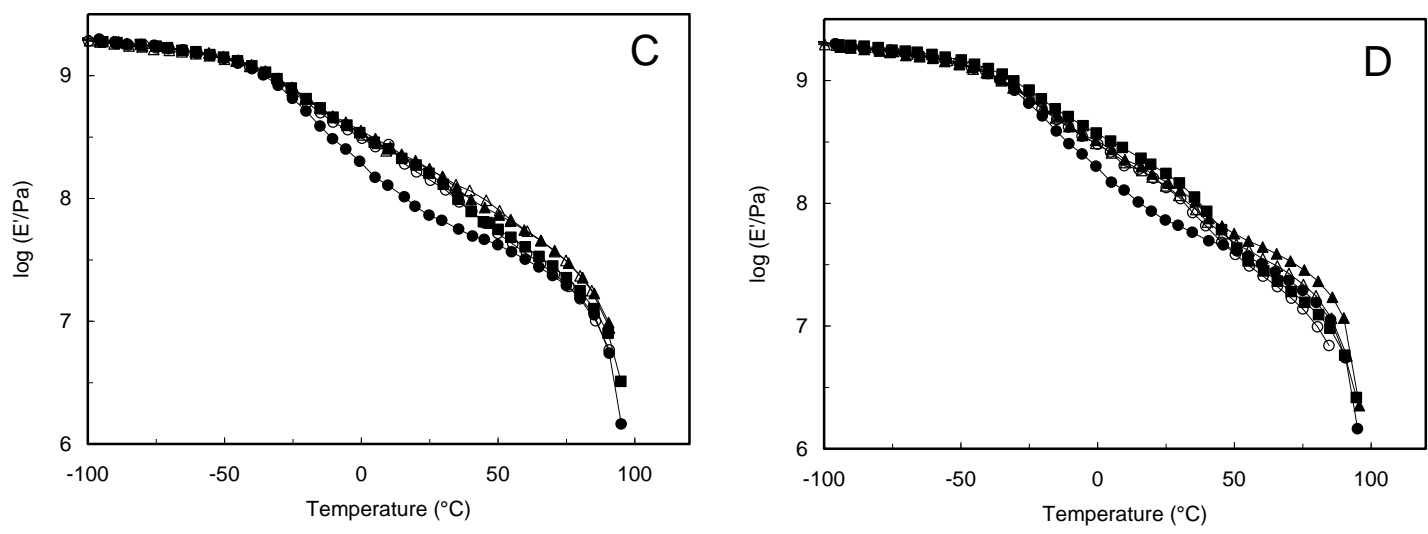

Figure 12: Evolution of the logarithm of the storage tensile modulus as a function of temperature for the neat LDPE matrix $(\bullet)$ and related nanocomposite films reinforced with 3 (A), 5 (B), 10 (C) and $15 \mathrm{wt} \%$ ramie cellulose whiskers: unmodified (०) and modified with hexanoyl chloride $(\boldsymbol{\Delta})$, lauroyl chloride $(\Delta)$ and stearoyl chloride $(\boldsymbol{\square})$. When adding ramie whiskers, the rubbery modulus slightly increases. This low but significant increase can be ascribed to a reinforcing effect of the cellulose whiskers and/or to the increase of the degree of crystallinity reported from DSC measurements. Surprisingly, no effect of the chemical grafting of aliphatic chains on the surface of the nanoparticles is observed. This is probably ascribed to two antagonist effects. Indeed, the chemical grafting should improve the dispersion of the filler within the polymeric matrix, but at the same time it decreases the possibility of inter-whiskers interactions. These interactions were reported to be the basis of the reinforcing effect for cellulose whiskers reinforced nanocomposites [21]. However, it is worth remember that for modified whiskers reinforced composites, the effective whisker content is lower than for its unmodified counterpart as previously emphasized.

In addition, most of the nanocomposite films display a two-steps modulus drop in the rubbery region of the LDPE matrix. Indeed, apart the one associated as for the neat matrix to the glass transition of LDPE, a high temperature modulus drop, around $35^{\circ} \mathrm{C}$ is observed. This new relaxation process is well identified through the maximum of the loss angle in Figure 13. The existence of the second tan delta peak could suggest that the main relaxation process, associated with $\mathrm{Tg}$ of the polymeric matrix, splits into two well-defined peaks. This splitting of the relaxation process could be ascribed to strong interactions between the functionalized cellulosic nanoparticles and the LDPE matrix. These interactions could lead to the formation of an interfacial layer surrounding the filler and which mobility is restricted compared to the bulk matrix. This phenomenon could be obviously emphasized because of the nanometric scale of the filler and omnispresence of the surface. However, if such an effect is at the origin of the splitting of the relaxation process, then the relative magnitude of the high temperature peak should increase when increasing the whiskers content, which was not observed. 
The second explanation is related to the possible cellulose whiskers-induced crystallization of the polymeric matrix. This explanation is highly speculative but the origin of this modulus drop remains mysterious. It is worth noting that the temperature of this second peak corresponds to the one of the melting endotherm observed for WRC18 in Figure 9.
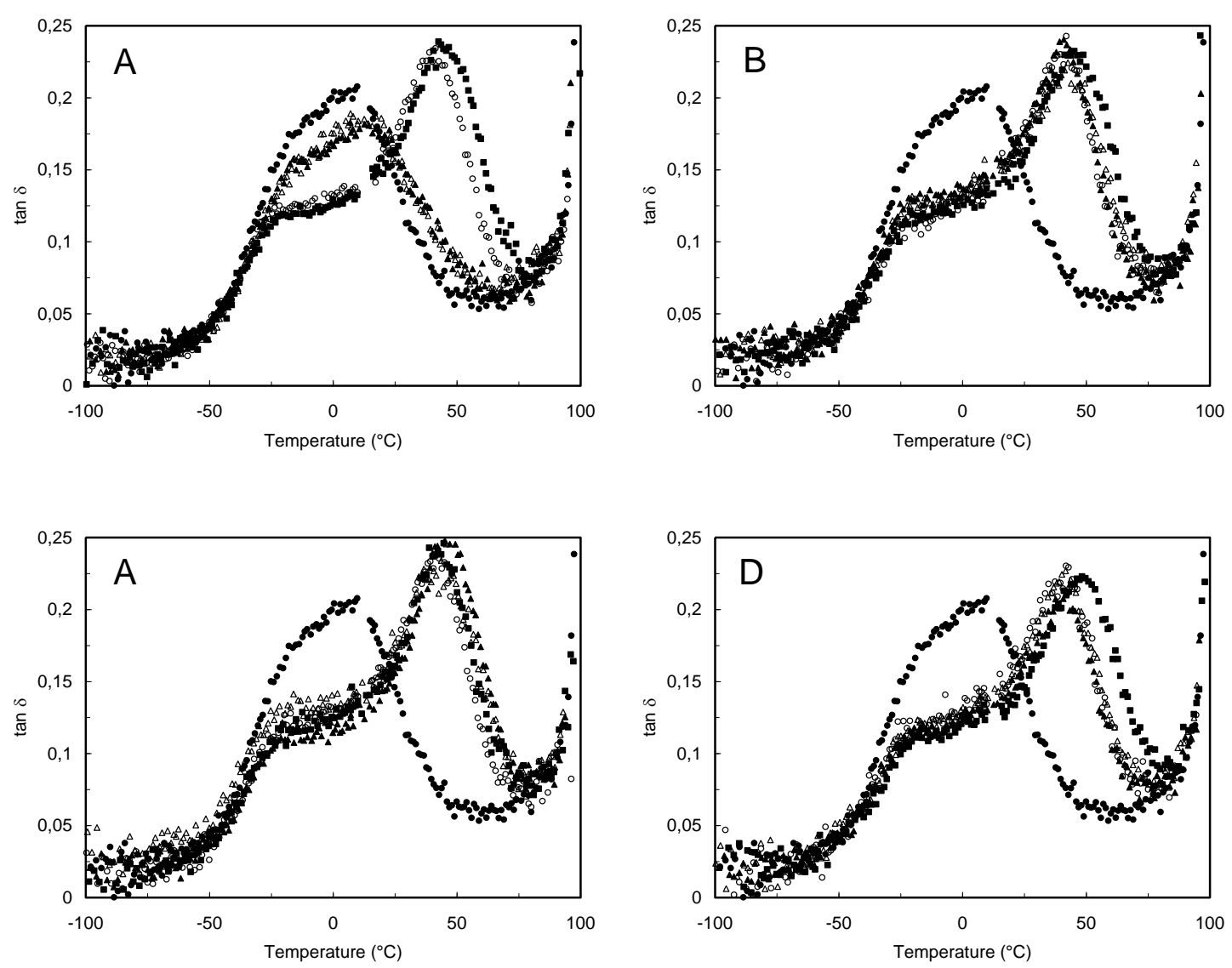

Figure 13: Evolution of the tangent of the loss angle ( $\tan \delta$ ) as a function of temperature for the neat LDPE matrix $(\bullet)$ and related nanocomposite films reinforced with 3 (A), 5 (B), 10

(C) and $15 \mathrm{wt} \%$ ramie cellulose whiskers: unmodified (O) and modified with hexanoyl chloride $(\boldsymbol{\Delta})$, lauroyl chloride $(\Delta)$ and stearoyl chloride $(\mathbf{\square})$.

Tensile tests were performed at room temperature. From the obtained stress-strain curves, the strength, tensile modulus and elongation at break for ramie cellulose whiskers reinforced LDPE were determined. Figure 14 shows the evolution of these parameters as a function of the whiskers content. A slight increase of the tensile modulus (Fig. 14B) and a slight decrease of the tensile strength (Fig. 14A) upon whiskers addition are observed. Again, no discriminating effect of the filler-matrix compatibilization is reported. However, as for other tests the comparison is difficult because both the effective whiskers content and the degree of crystallinity of the specimen differ depending on the chemical modification of the filler. 

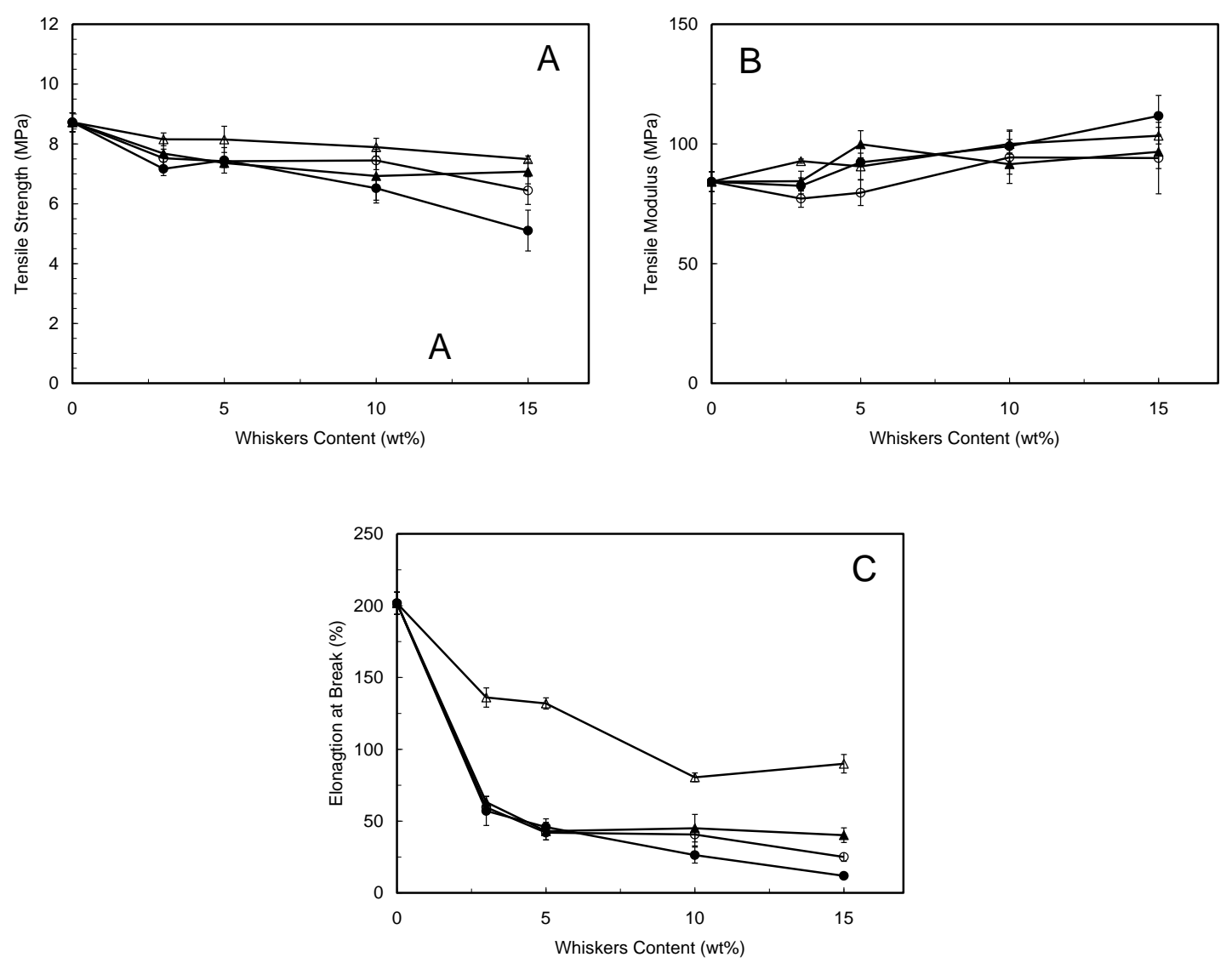

Figure 14: Evolution of the tensile strength (A), tensile modulus (B) and elongation at break (C) as a function of the ramie cellulose whiskers content for nanocomposite films reinforced with unmodified $(\bullet)$ and modified with hexanoyl chloride $(\circ)$, lauroyl chloride $(\boldsymbol{\Delta})$ and stearoyl chloride $(\Delta)$ whiskers. The lines serve to guide the eyes.

The elongation at break decreases upon whiskers addition as expected and tends to roughly stabilize at high filler loading (Fig. 14C). However, an interesting feature is observed. When the grafted chains are sufficiently long (C18-grafted nanoparticles), the elongation at break is systematically much higher for a given composition than for other samples. It could be due to a possible co-crystallization between covalently linked chains at the surface of the nanoparticles with those from the matrix, but this co-crystallization was not evidenced from other measurements. Entanglements between grafted chains and macromolecular chains from the polymeric matrix are highly unlikely because of the low molecular weight of the grafted moieties. The most probable explanation could be the highest dispersion level of cellulose whiskers chemically modified with C18 chains.

\section{Conclusions}

The surface of cellulose nanocrystals or whiskers prepared by acid hydrolysis of ramie fibers was chemically modified using organic acid chloride aliphatic chains of different sizes, namely hexanoyl chloride, lauroyl chloride and stearoyl chloride. The evidence of occurrence of chemical modification was evidenced by FTIR and X-ray photoelectron spectroscopies. It 
was checked from X-ray diffraction analysis that the initial crystalline structure was preserved. The reduction of the polar character as determined by contact angle measurements showed that the surface chemical modification allowed enhancing the nonpolar nature of original cellulose nanocrystals, thereby allowing the use of nonpolar polymers as matrices for the processing of nanocomposite materials. Most of the studies reported in the literature with cellulose whiskers reinforced nanocomposites involve casting/evaporation processing technique. In the present study, we used a highly hydrophobic commodity plastic which is not soluble in common solvents or available in the form of latex, viz. low density polyethylene (LDPE). Indeed, almost no study of the processing of cellulose whiskers reinforced nanocomposites using industrial techniques such as extrusion has been reported. Both unmodified and functionalized nanoparticles were extruded with LDPE to prepare nanocomposite materials. The homogeneity of the ensuing nanocomposites was found to increase with the length of the grafted chains. The thermomechanical properties of processed nanocomposites were studied by DSC, DMA and tensile tests. A significant improvement in terms of elongation at break was observed when sufficiently long chains were grafted on the surface of the nanoparticles.

\section{Acknowledgments}

The authors thank CAPES (Brazil) for financial support.

\section{References}

1 Bledzki AK, Gassan J. Prog Polym Sci 1999;24:221-274; Eichhorn SJ, Baillie CA, Zafeiropoulos N, Mwaikambo LY, Ansell MP, Dufresne A, Entwistle KM, HerreraFranco PJ, Escamilla GC, Groom L, Hugues M, Hill C, Rials TG, Wild PM. J Mater Sci 2001;36:2107-2131; Eco-composites. A special issue of Compos Sci Technol 2003;63, 114 pp., a collection of 14 publications all dedicated to cellulose-based composite materials; Mohanty AK, Misra M, Drzal LT. Natural fibers, biopolymers, and biocomposites, Boca Raton: CRC Press, 2005; Jacob John M, Thomas S. Carbohydr Polym 2008;71:343-364.

2 Sakurada I, Nukushina Y, Ito T. J Polym Sci 1962;57:651-660.

3 Tashiro K, Kobayashi M. Polymer 1991;32:1516-1526.

4 Šturcová A, Davies GR, Eichhorn SJ. Biomacromolecules 2005;6:1055-1061.

5 Anglès MN, Dufresne A. Macromolecules 2000;33:8344-8353; Mathew AP, Dufresne A. Biomacromolecules 2002;3:609-617; Orts WJ, Shey J, Imam SH, Glenn GM, Guttman ME, Revol JF. J Polym Env 2005;13:301-306, Kvien I, Sugiyama J, Votrubec M, Oksman K. J Mater Sci 2007;42:8163-8171; Azizi Samir MAS, Alloin F, Sanchez JY, Dufresne A. Polymer 2004;45:4033-4041; Azizi Samir MAS, Alloin F, Gorecki W, Sanchez JY, Dufresne A. J Phys Chem B 2004;108:10845-10852; Azizi Samir MAS, Montero Mateos 
A, Alloin F, Sanchez JY, Dufresne A. Electrochim Acta 2004;49:4667-4677; Azizi Samir MAS, Chazeau L, Alloin F, Cavaillé JY, Dufresne A, Sanchez JY. Electrochim Acta 2005;50:3897-3903; Azizi Samir MAS. Alloin F, Dufresne A. Compos Interfaces 2006;13:545-559; Zimmermann T, Pöhler E, Geiger T. Adv Eng Mat 2004;6:754-761; Zimmermann T, Pöhler E, Schwaller P. Adv Eng Mat 2005;7:1156-1161; Choi YJ, Simonsen J. J Nanosci Nanotechnol 2006;6:633-639.

6 Favier V, Canova GR, Cavaillé JY, Chanzy H, Dufresne A, Gauthier C. Polym Adv Technol 1995;6:351-355; Helbert W, Cavaillé JY, Dufresne A. Polym Compos 1996;17:604-611; Dufresne A, Cavaillé JY, Helbert W. Polym Compos 1997;18:198-210; Dubief D, Samain E, Dufresne A. Macromolecules 1999;32:5765-5771; Dufresne A, Kellerhals MB, Witholt B. Macromolecules 1999;32:7396-7401; Dufresne A. Compos Interfaces 2000;7:53-67; Chazeau L, Cavaillé JY, Canova GR, Dendievel R, Boutherin B. J Appl Polym Sci 1999;71:1797-1808; Chazeau L, Cavaillé JY, Terech P. Polymer 1999;40:5333-5344; Chazeau L, Paillet M, Cavaillé JY. J Polym Sci B: Polym Phys 1999;37:2151-2164; Chazeau L, Cavaillé JY, Perez J. J Polym Sci B: Polym Phys 2000;38:383-392; Matos Ruiz M, Cavaillé JY, Dufresne A, Graillat C, Gérard JF. Macromol Symp 2001;169:211-222; Garcia de Rodriguez NL, Thielemans W, Dufresne A. Cellulose 2006;13:261-270.

7 Heux L, Chauve G, Bonini C Langmuir 2000;16:8210-8212; Kvien I, Bjom ST, Oksman K. Biomacromolecules 2005;6:3160-3165.

8 Goussé C, Chanzy H, Exoffier G, Soubeyrand L, Fleury E. Polymer 2002;43:2645-2651; Gopalan Nair K, Dufresne A, Gandini A, Belgacem MN. Biomacromolecules 2003;4:1835-1842; Angellier H, Molina-Boisseau S, Belgacem MN, Dufresne A. Langmuir 2005;21:2425-2433.

9 Azizi Samir MAS, Alloin F, Sanchez JY, El Kissi N, Dufresne A. Macromolecules 2004;37:386-1393; Marcovich NE, Auad ML, Belessi NE, Nutt SR, Aranguren MI. J Mater Res 2006;21:870-881; van den Berg O, Capadona JR, Weder C. Biomacromolecules 2007;18:1353-1357.

10 Nakagaito AN, Yano H. Appl Phys A 2004;78:547-552; Nakagaito AN, Iwamoto S, Yano H. Appl Phys A 2005;80:155-159; Nakagaito AN, Yano H. Cellulose 2008;15:323331; Shimazaki Y, Miyazaki Y, Takezawa Y, Nogi M, Abe K, Ifuku S, Yano H Biomacromolecules 2007;8:2976-2978; Yano H, Sugiyama J, Nakagaito AN, Nogi M, Matsuura T, Hikita M, Handa K. Adv Mat 2005;17:153-155; Nogi M, Handa K, Nakagaito AN, Yano H. Appl Phys Lett 2005;87:243110; Iwamoto S, Abe K, Yano H. Biomacromolecules 2008;9:1022-1026; Henriksson M, Berglund LA. J Appl Polym Sci 2007;106:2817-2824. 
11 Oksman K, Mathew AP, Bondeson D, Kvien I. Compos Sci Technol 2006;66:2776-2784.

12 Bondenson D, Oksman K. Composites: Part A 2007;38:2486-2492.

13 Thielemans W, Belgacem MN, Dufresne A. Langmuir 2006;22:4804-4810.

14 D.K. Owens and R.C. Wendt, J. Appl. Polym. Sci., 1969, 13, 1741-1747.

15 Habibi Y, Goffin AL, Schiltz N, Duquesne E, Dubois P, Dufresne A. J Mater Chem 2008;18:5002-5010.

16 Freire CSR, Silvestre AJ.D, Pascal Neto C, Gandini A, Fardim P, Holmbom B. J Coll Interface Sci 2006;301:205-209.

17 Vaca-Garcia C, Borredon ME, Gaseta A. Cellulose 2001;8:225-231.

18 Vaca-Garcia C, Borredon ME. Bioresource Technology 1999;70:135-142; Thiebaud S, Borredon ME. Bioresource Technology 1995;52:169-173.

19 Habibi Y, Dufresne A. Biomacromolecules 2008;9:1974-1980.

20 Ferreira FC, Curvelo AAS, Mattoso LHC. J Appl Polym Sc 2003;89:2957-2965.

21 Azizi Samir MAS, Alloin F, Dufresne A. Biomacromolecules 2005;6:612-626. 